Original Research

\title{
Transvaginal PVDF-mesh for cystocele repair: A cohort study
}

\author{
Dimitri Barski ${ }^{\text {a, * }}$, Christian Arndt ${ }^{a}$, Holger Gerullis ${ }^{b}$, Jin Yang ${ }^{\mathrm{c}}$, Mihaly Boros ${ }^{\mathrm{d}}$, \\ Thomas Otto ${ }^{a}$, Hans-Christian Kolberg ${ }^{\mathrm{e}}$ \\ a Department of Urology, Lukas Hospital, Neuss, Germany \\ ${ }^{\mathrm{b}}$ University Hospital for Urology, Klinikum Oldenburg, School of Medicine and Health Sciences, Carl von Ossietzky University Oldenburg, Germany \\ ${ }^{c}$ Department of Urology, Affiliated Hospital of Chengdu University, Chengdu, China \\ d Department of Experimental Surgery, University of Szeged, Hungary \\ e Department of Gynecology, Marienhospital Bottrop, Germany
}

\section{H I G H L I G H T S}

- PVDF- mesh is feasible for transvaginal application for cystocele repair.

- $15 \%$ of patients presented any kind of complications and 8.8\% needed a resurgery for SUI or POP during the follow-up.

- Improvement of quality of life was reported by $87.5 \%$ according the PGI-I.

- The preliminary results support the initiation of prospective trial and registry according to IDEAL.

\section{A R T I C L E I N F O}

\section{Article history:}

Received 31 January 2017

Accepted 6 February 2017

Available online 10 February 2017

\section{Keywords:}

Anterior mesh

Alloplastic material

Biocompatibility

Vaginal wall prolapse

Cystocele

IDEAL

\begin{abstract}
A B S T R A C T
Introduction: Optimized biocompatibility of new materials is a major requirement for transvaginal meshes for pelvic organ prolapse (POP) repair. Polyvinylidene fluoride (PVDF) presented good characteristics in prior animal experiments and clinical use in humans.

Methods: Between 01/2012 and 04/2016 37 women underwent transvaginal repair of symptomatic prolapse of the anterior vaginal wall (cystocele) with PVDF-mesh in a single institution. A chart review for recurrence, continence, peri- and early postoperative complications was performed. Referring practitioners were interviewed by telephone and mail. Additionally patient reported outcome and satisfaction were measured by Patient Global Improvement Inventory (PGI-I) scale.

Results: 34 women were eligible for a mean follow up of 19 months. The functional outcome improved significantly. One symptomatic vault prolapse (2.9\%) and two reoperations for incontinence (5.9\%) occurred. Two mesh exposures (5.9\%) occurred and were treated conservatively. No other severe complications were registered. $87.5 \%$ of treated women felt very much better or much better and would undergo the surgery again. A cohort study including development steps in accordance with the IDEAL system is presented.

Conclusions: For the first time we report on effectivity and safety of transvaginal application of PVDFmesh in real-life practice. A prospective long-term evaluation in a registry is justified.
\end{abstract}

๑ 2017 IJS Publishing Group Ltd. Published by Elsevier Ltd. All rights reserved.

\section{Introduction}

Recently there has been a rising interest in new surgical

\footnotetext{
* Corresponding author. Department of Urology, Lukas Hospital Neuss, Preussenstr. 84, 41464 Neuss, Germany.

E-mail addresses: dbarski@lukasneuss.de (D. Barski), carndt@lukasneuss.de (C. Arndt), holger.gerullis@gmx.net (H. Gerullis), 5458839@qq.com (J. Yang), boros.mihaly@med.u-szeged.hu (M. Boros), thomas_otto@lukasneuss.de (T. Otto), hans-christian.kolberg@mhb-bottrop.de (H.-C. Kolberg).
}

techniques and materials that may improve outcome after cystocele repair. Standardised trocar-guided meshes are applied in prolapse surgery to reduce the recurrence of prolapse for decades. Polypropylene (PP) meshes (Type 1, Amid-classification) are the most used [1]. New modifications with light-weight meshes, coatings and partly resorbable materials have been proposed lately. However, there are no standards and less evidence on relevant biological and physical parameters of materials [2]. Due to insufficient medical product controls a lot of me-too meshes got approval without the provision of studies first. Food and Drug 
Administration (FDA) changed the requirements for mesh approval due to the number of serious complications related to mesh insertion. In January 2016 an FDA amendment was released, which requires studies and clinical data before the application of transvaginal meshes [3]. Postmarket-surveillance is required by the European regulatory authorities. Alloplastic materials potentially add to the complication profile the aspects of trauma of insertion, foreign body reaction to the implant in terms of inflammation, infection and/or rejection, and the stability of the prosthesis over time [4]. The rate of mesh-related complications after the implantation of transvaginal mesh is about $15-25 \%$ and especially mesh erosion up to $10 \%[5,6]$. Other complications are obstruction, de novo urge, chronic pain, dyspareunia and mesh erosion [7]. Biocompatibility is an important requirement for the perfect ingrowth of the material. It is determined by the foreign body reaction and inflammation and depends on different parameters like type of polymer, pore size, material weight and others [8,9]. An optimized surgical mesh permits the transmigration and localisation of immune cells and prevents adherence and inflammation if directly exposed to visceral organs, vessels or nerves.

In reaction to FDA warnings, our international scientific collaboration group has recently developed and concluded preliminary studies in order to investigate and improve biocompatibility of surgical meshes [3,9-11]. Our entire innovative approach has been conducted following the five stage IDEAL-D method of surgical innovations (Idea, Development, Exploration, Assessment, and Long-term study of Device) with the aim of comparability and reproducibility at every single step of development [12,13]. Experimental studies have been conducted first and can be assigned to preclinical stage 0 according to IDEAL-D [13]. For the first time a validated in vitro test system to compare the biocompatibility of different meshes was developed [9]. Series of commonly used meshes were incubated with patient tissue culture (muscle, connective tissue and endothelium) and the adhesion and ingrowth of tissue over time were assessed. The score system was then validated in a longterm animal [11]. Different meshes were inserted intraperitoneally and as a fascia and muscle onlay in a sheep and were explanted after hours, 3, 6, 12 and 24 mos. Complications were evaluated and connective tissue and inflammatory reaction were examined histologically. The study revealed comparable ranking characteristics at every time point after explantation. The in vivo performance of these meshes in a sheep model was predictable with a previously developed in vitro test system. The early inflammatory reaction determined the outcome and mesh biocompatibility [10].

After preliminary positive results on PVDF-mesh in vitro and in a longterm animal study consecutive human application is presented in the current study according to the first stage of IDEAL-D. The aim of the study is to proof the efficacy and safety of a new mesh material in a real-life practice. Additional focus of the study was on the Patient-Reported Outcome (PRO).

\section{Methods}

\subsection{Patients}

The study was carried out in accordance with the 1964 Helsinki Declaration and its later amendments. An ethics vote has not been applied for due to the retrospective design. 37 women were retrospectively enrolled who underwent transvaginal anterior PVDF mesh repair for POP between 2012 and 2016 in a single center cohort study(Marienhospital Bottrop, Germany). All women gave their signed informed consent for participation in this study. Study methods and definitions were applied according to recommendations by the International Urogynecological Association (IUGA) and the International Continence Society (ICS) [4,14]. Women in reproductive age, planning of pregnancy were excluded for mesh usage. Women with uterovaginal prolapse were recommended to concomitant hysterectomy. The study group included parous women with symptomatic grade II-IV anterior/apical prolapse based on Pelvic Organ Prolapse Quantification (POP-Q). All grade II cystoceles were recurrent and symptomatic after previous surgery for prolapse. Risk factors like recurrent or high grade cystocele, obesity, chronic obstructive pulmonary disease, heavy physical labour, previous pelvic floor surgeries and preference of the patients were considered in cases of vaginal mesh insertion [5]. PVDF-mesh was used due to beneficial material characteristics found in previous studies [9-11]. From January 2012 through April 2016, patients were screened by the participating surgeons for prolapse of the anterior vaginal wall after referral by their Gynecologist. All patients experienced an unsuccessful conservative treatment prior to operation. Exclusion criteria were previous cancer of any pelvic organ, previous mesh implantation at the operation site, infection at the operation site, chemo- or immunological therapy during the last three months, pregnancy or wish for child. All patients selected for the surgery underwent preoperative and postoperative clinical examination and pelvic floor ultrasound by the referring Gynecologist. Table 1 presents the clinical characteristics of the study group. Additionally the study was registered at German Clinical Trials Register (DRKS), registration number DRKS00011264 and Research Registry, registration number researchregistry2097.

\subsection{Surgical procedure}

All surgeries were performed by one experienced Urogynecologist (HCK). The surgical procedures were standardised before initiation of the study. All mesh procedures involved use of the PVDF mesh (DynaMesh ${ }^{\circledR}$-PR4, FEG Textiltechnik, Germany) (Fig. 1). The material is a pre-cut non-absorbable monofilament soft PVDF mesh $(6 \times 4 \mathrm{~cm})$ with four arms. This procedure may be performed under spinal or general anesthesia. All patients had an intravenous perioperative antibiotic prophylaxis. All patients were placed in the lithotomy position with thighs flexed at approximately $90^{\circ}$. After cleaning the entire surgical area with antiseptic, a suprapubic catheter is placed. The anterior vaginal wall is cut and the cystocele

Table 1

Baseline characteristics of 37 patients undergoing cystocele repair with PVDF-mesh.

\begin{tabular}{ll}
\hline Mean age, years \pm SD (range) & $66( \pm 11.5)$ \\
Parity & 2 \\
$\quad$ Median & $0-5$ \\
Range & $1(2.7)$ \\
Cesarean deliveries, no. of patients (\%) & $5(13.5)$ \\
Current smokers, no. of patients (\%) & $27( \pm 4.1)$ \\
Body mass index, mean \pm SD (range) & $33(89.2)$ \\
Menopause, no. of patients (\%) & $11(29.7)$ \\
Current use of hormone therapy, no. of patients (\%) & $14(37.8)$ \\
Risk factors (diabetes mellitus, immunosuppression, & \\
recurrent UTIs, PVR $>100$ ml), no. of patients (\%) & $8(21.6)$ \\
Previous surgery for cystocele, no. of patients (\%) & \\
Prior pelvic surgery, no. of patients (\%) & $13(35.1)$ \\
hysterectomy & $4(10.8)$ \\
sacrospinal fixation & $1(2.7)$ \\
suburethral tape & $3 \pm 0.4(2-4)$ \\
Mean POP-Q grade, n \pm SD (range) & $6(16.2)$ \\
Grade II, n (\%) & $26(70.3)$ \\
Grade III, n (\%) & $5(13.5)$ \\
Grade IV, n (\%) & \\
Symptoms, no. of patients (\%) & $29(78.4)$ \\
vaginal bulge & $13(40.5)$ \\
episodes of incontinence & $3(8.1)$ \\
pelvic pain &
\end{tabular}




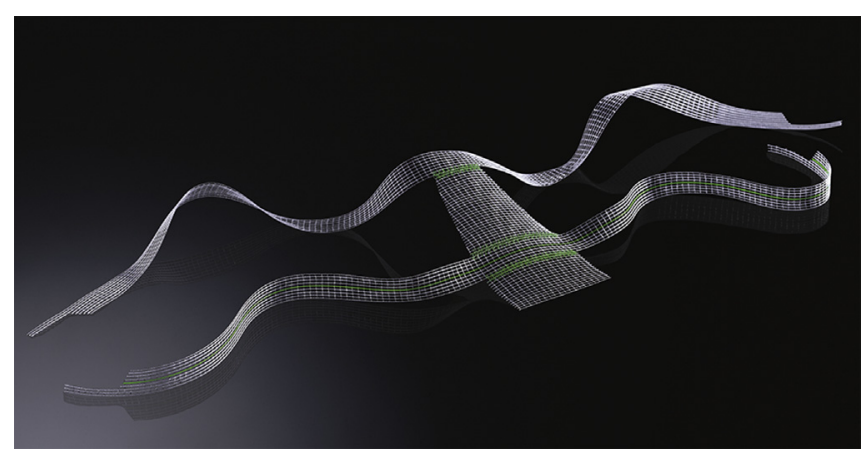

Fig. 1. PVDF-mesh (Dynamesh $^{\circledR}$, PR4), with permission of FEG Textiltechnik (Germany).

is prepared carefully, so that a full thickness vaginal wall and perivaginal fat tissue are exposed. At the superior part the dissection is extended over the pelvic floor in order to expose the access to obturator foramen on both sides. The preparation is done bluntly to palpate spina ischiadica or arcus tendineus on both sides. Skin incisions are done at the anteromedial margin of the foramen oburatorium and $2 \mathrm{~cm}$ posterior and $1 \mathrm{~cm}$ lateral of the site. Trocar is inserted through foramen obturatorium, over the insertion of arcus tendineus and is lead through colpotomy. The mesh arms are introduced retrograde with trocar on both sides. After finding the optimal tension- and wrinkle-free position the mesh arms are fixed apical with interrupted sutures of Vicryl. The colpotomy is closed with continuous Vicryl suture (Fig. 2). Concomitant procedures were performed if necessary, including hysterectomy in $20 \mathrm{pa}-$ tients, sacrospinal fixation in 18 patients, sacrocolpopexy in 3 patients and posterior vaginal wall repair in 20 patients. During the whole series we made several changes to our operative procedure due to experience and mesh modification:

1. From case 6 onwards a suprapubic tube was placed during the surgery to enable a controlled postoperative bladder training for the patients. All but one of previous cases needed a recatheterisation due to prolonged postoperative voiding difficulties. Additionally a postoperative vaginal ultrasound examination was performed in order to rule out a hematoma.

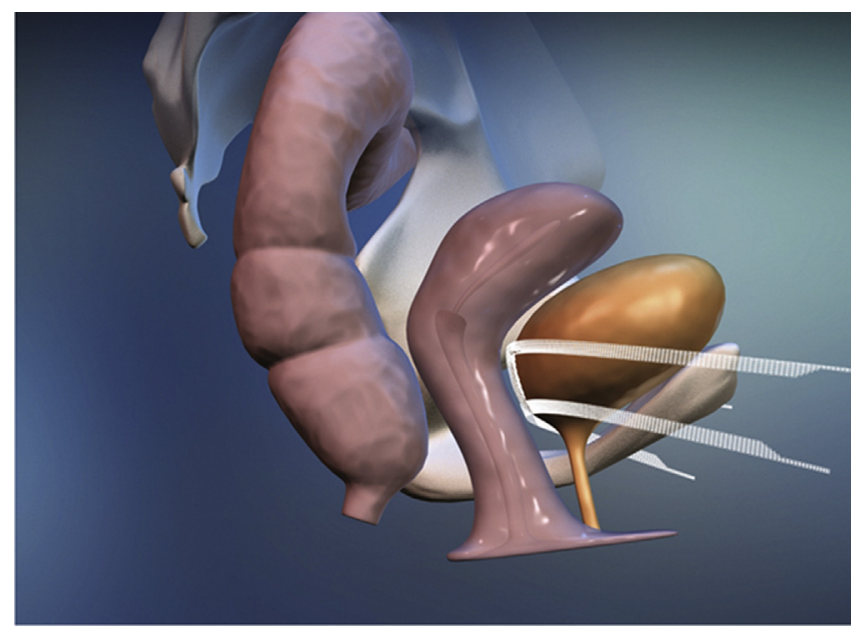

Fig. 2. Cystocele repair with anterior vaginal wall insertion of a PVDF-mesh (Dynamesh $^{\circledR}$, PR4), with permission of FEG Textiltechnik (Germany).
2. After case 11 additional apical fixation of the mesh arms with interrupted sutures of Vicryl was done to prevent the moving or shrinkage of the mesh.

3. After case 16 additional fixation of the apical vaginal wall by sacrospinal fixation or sacrocolpexy was performed in cases of apical prolapse in order to prevent recurrences in this compartment.

4. From case 35 onward a new modified PVDF mesh (DynaMesh ${ }^{\circledR}$ PR4 visible, FEG Textiltechnik, Germany) was used. The new mesh is MRI-visible, which enables a better postoperative control and has an additional apical part for a better fixation. However, MRI-studies are not presented in this study.

\subsection{Follow up}

Postmenopausal patients received postoperative topical estrogen cream treatment. Patients stayed normally for one week in hospital as standard care to train the bladder emptying. The suprapubic catheter was removed before discharge after 5-7 days and measured post-void residual urine volume (PVR) $<100 \mathrm{ml}$. All patients underwent clinical examination and pelvic floor ultrasound before discharge in the hospital. Medical records of women included in the study were reviewed for preoperative characteristics including risk factors (body mass index, smoking, number of deliveries, diabetes etc.), postoperative complications according to Clavien-Dindo classification [15] and ICS/IUGA classification [4]. The range of $15-25 \%$ mesh-related complications was defined to be met by the clinical outcome of this study $[5,6]$. At follow up the patients were interviewed and examined by the referring Gynecologists. The Gynecologists were interviewed by DB and CA about the symptoms, complications and reoperations of the patients at follow-up.

\subsection{Patient reported outcome}

The subjective measure of women's satisfaction was evaluated by Patient Global Improvement Inventory (PGI-I) scale (range 1-7, very much better to very much worse), which has been validated as a measurement of global improvement after prolapse surgery previously [16,17]. For the definition of complete success, we used a simple yes/no question: "If you had to undergo surgery all over again, would you still do it?" [17]. Additionally, treated women were asked for postoperative complications and symptoms (Fig. 3).

\subsection{Statistical analysis}

The data were documented into Microsoft Excel software and then transferred into an GraphPadPrism6.0 (Graphpad Software, Inc.) data bank for statistical analysis. Descriptive statistic was used for evaluation of complication rates and analysis of the questionnaire. Continuous data were checked for normality of distribution before choosing between parametric and non parametric tests. The results were presented as medians with range or means with standard deviation (SD) in case of normal distribution. The $t$-test was utilized for comparison of the complications between the groups with or without concomitant operations and for the group with surgical modification by apical mesh fixation. A p value below 0.05 was considered statistically significant.

\section{Results}

Between January 2012 and April 201637 patients underwent POP surgery with Dynamesh ${ }^{\circledR}$ PR4 for symptomatic cystocele in a single institution. More than $80 \%$ presented $\geq$ grade 3 cystocele and 
1). Check the number that best describes how your post-operative condition is now, compared with how it was before you had the surgery.

Very much better

Much better

A little better

No change

A little worse

Much worse

Very much worse

2). If you had to undergo surgery all over again, would you still do it?

$$
\text { Yes }
$$

No

3). Did you suffer under postoperative complications?
No
Yes
Please explain:

4). Did you undergo a re-surgery for pelvic organ prolapse?

No
Yes
Please explain:

Additional comments:

Fig. 3. Postoperative patient questionnaire with PGI-I scale [17].

$21.6 \%$ underwent a previous surgery for cystocele. Vaginal bulge symptoms were presented by 29 patients (78\%) and 13 patients (40.5\%) suffered from episodes of incontinence at least once a week (Table 1). Perioperative data is presented in Table 2. The mean follow up was 19 months. Three patients were lost to follow up, as they did not refer to their Gynecologist after the operation. In 86\% of patients concomitant operations (hysterectomy, sacrospinous fixation, sacrocolpopexy, posterior repair) were performed. No

Table 2

Surgical and perioperative characteristics.

\begin{tabular}{ll}
\hline General anesthesia, no. of patients (\%) & $35(94.6)$ \\
Regional anesthesia, no. of patients (\%) & $2(5.4)$ \\
Operation time, mean (min) & $70( \pm 18.2)$ \\
$\begin{array}{l}\text { Operation time (anterior mesh only), median (min) } \\
\text { Concomitant procedures, no. of patients (\%) }\end{array}$ & $40(38-40)$ \\
$\quad$ hysterectomy & $21(56.7)$ \\
sacrospinal fixation & $18(48.6)$ \\
abdominal sacrocolpopexy & $3(8.1)$ \\
posterior prolapse repair & $20(54)$ \\
Complications during surgery, no. of patients (\%) & \\
rectum lesion (Clavien-Dindo Grade I, ICS/IUGA: 5Aa/T1/S2) & $1(3.3)$ \\
$\quad$ blood loss in excess of 500 ml & 0 \\
Hospital stay, median (days) & $7(5-14)$ \\
\hline
\end{tabular}

statistical significance for the occurrence of complications was detected for patients with concomitant operations. The efficacy of apical mesh fixation was not significant. Complications are presented in Table 3. Overall 15\% of patients presented any kind of complications and $8.8 \%$ needed a resurgery for SUI or POP during the follow-up. Assuming the three lost to follow up patients to have a bad outcome, overall $22 \%$ would suffer any kind of complications and $16 \%$ would need a resurgery for SUI or POP during the followup. In one case a rectal lesion $\leq 1 \mathrm{~cm}$ occurred during concomitant posterior wall repair and was treated conservatively. No intraoperative problems or complications associated with mesh insertion were observed. There were three cases of vaginal wall hematoma (8.8\%, Clavien-Dindo I) during the hospital stay. Two patients with transient postoperative voiding dysfunction and PVR $>100 \mathrm{ml}$ were discharged with indwelling catheter (5.9\%, ClavienDindo II). There were two cases of mesh exposure (5.9\%). In the first case there was a persisting postoperative mesh exposure due to wound dehiscence and hematoma, a closure of the vaginal wall under anesthesia was needed four weeks after the initial mesh insertion (Clavien-Dindo III). Another case of vaginal mesh exposure with temporary bleeding one year after initial surgery was treated conservatively with topical estrogen. The symptoms were relieved without a surgical intervention. Three patients suffered from de novo incontinence (8.8\%), in two cases a suburethral tape was inserted over time (5.9\%, Clavien-Dindo III). One patient complained from prolapse and presented POP-Q grade III apical prolapse recurrence 6 months after anterior mesh application and concomitant sacrospinal fixation. A repair with sacrocolopexy is planned (2.9\%, Clavien-Dindo III). One patient presented with vaginal bleeding and pelvic pain and underwent hysterectomy two years after the initial surgery. No other reoperations occurred during the follow-up. No mesh resections or explantations were necessary till the date of last follow up, which is September 2016. From our collective $87.5 \%$ of treated women felt very much better or much better after the surgery and would undergo the surgery all over again. However, at least two patients were not satisfied as their symptoms did not change or got a little worse after the operation, one woman suffered under recurrent prolapse and another woman claimed persisting incontinence.

\section{Discussion}

Meshes are used for decades for the reinforcement of pelvic floor tissue. However, meshes probably should not been used as first choice, but there are indications in case of higher risk of recurrence $[2,5]$. The recurrence rates could be reduced significantly but for the price of an increased inflammatory and fibrotic tissue reaction to the implant. Current examinations show the importance of acute inflammatory and immune responses for the integration of mesh into the surrounding tissue $[10,18]$. The studies aim to engineer a material which holds shape over time and reduces the inflammatory host reaction. Biomechanical characteristics like effective porosity and material weight are crucial for the mesh performance in host. According to the inflammatory reaction and biomechanical characteristics new classification of meshes has been proposed recently by Klosterhalfen and Klinge [18].

Polyvinylidene fluoride (PVDF) is a polymer with improved textile and biological properties [19,20]. In comparison to other materials like polyethylene-terephtalat (PET), PVDF is more resistant to hydrolysis and degradation. Furthermore, ageing does not increase the stiffness, evidently seen in polypropylene (PP). A German group tested already 2002 PVDF mesh for abdominal wall reconstruction in rats [19]. These results were supported and standardised by our group in in vitro and large animal studies showing good biocompatibility of PVDF [11]. In the preclinical 
Table 3

Postoperative complications.

\begin{tabular}{|c|c|c|c|}
\hline Postoperative complications & & Clavien-Dindo & ICS/IUGA Classification \\
\hline Follow-up in mos, mean \pm SD (range) & $19 \pm 8.5(2-48)$ & & \\
\hline \multicolumn{4}{|c|}{ Complications during hospital stay, no. of patients (\%) } \\
\hline vaginal hematoma & $3(8.8)$ & I & $7 \mathrm{~A} / \mathrm{S} 3 / \mathrm{S} 4$ \\
\hline catheter after hospital stay & $2(5.9)$ & II & $4 \mathrm{~B} / \mathrm{T} 2$ \\
\hline urinary tract infection & $2(5.9)$ & II & $4 \mathrm{~B} / \mathrm{T} 2$ \\
\hline \multicolumn{4}{|c|}{ Complications related to surgical procedure between hospital stay and follow up, no. of patients (\%) } \\
\hline surgery for stress urinary incontinence & $2(5.9)$ & III & $4 \mathrm{~B} / \mathrm{T} 2$ \\
\hline surgery for prolapse recurrence & $1(2.9)$ & III & nd \\
\hline mesh exposure & $2(5.9)$ & I & 2B/T1/S3 \\
\hline pelvic pain & $1(2.9)$ & I & 6B/T3/S5 \\
\hline
\end{tabular}

studies we found beneficial characteristics of PVDF-polymer with reduced inflammatory reaction and scarring at the implantation site $[9,11]$. Several studies on PVDF mesh application for surgical hernia repair are available. An Italian study proved Dynamesh ${ }^{\circledR}$ IPOM to be a safe and effective mesh for the laparoscopic repair of incisional hernia in a small prospective trial [21]. Another study analysed 181 patients from the National Danish Health Registry after Dynamesh ${ }^{\circledR}$ application in laparoscopic ventral hernia repair. They reported $6 \%$ risk of mesh-related reoperation and chronic pain in $19 \%$, however patient satisfaction was high after 34 months follow-up [22]. To our knowledge there are only two German studies reporting on small series of PVDF application for the reconstruction of pelvic floor. Joukhadar and colleagues modified laparoscopic bilateral sacropexy (MLBS) in 10 patients using a MRIvisible PVDF mesh implant and reported favourable anatomical and functional outcome after average follow-up of 7.4 months [23]. This study can be assigned to stage 1 of IDEAL-system. Another singlecenter randomized prospective clinical trial presented data on laparoscopic bilateral fixation of the vagina/cervix to the iliopectineal ligaments via a PVDF-mesh in 44 patients versus 41 standard laparoscopic sacropexies with PVDF-mesh [24]. The intermediateterm follow-up (21.8 months for pectopexy and 19.5 months for sacropexy) showed no mesh-related adverse events with high overall satisfaction (>95\%). This study can be assigned to stage 1 of IDEAL-system, however we present large case series with detailed information on surgical technique modification and postoperative follow-up.

Our study presents the first transvaginal application of the PVDF-mesh for cystocele repair. Most of the selected patients presented a symptomatic high grade cystocele $(83.8 \% \geq$ grade III cystocele, $78 \%$ with bulge symptoms) or recurrence after initial anterior vaginal colporrhaphy (21.6\%). All patients with grade II POP were recurrent and had possible risk factors for reoperation, like diabetes, obesity and multiple previous surgeries. A suprapubic tube was placed during the operation and was removed after successful bladder training during the hospital stay. Voiding dysfunction is a common transient complication of transvaginal anterior mesh [5,6]. Altman et al. reported about transient bladder emptying difficulties during the hospital stay in $8 \%$ of patients after mesh repair [25]. A current German trial revealed that patients cystocele repair using mesh are at increased risk of developing postoperative voiding dysfunction, so that postoperative suprapubic bladder drainage is beneficial [26]. Due to this management only 2 patients needed the catheter over the time of hospital stay in our series. The reason for voiding dysfunction were postoperative hematoma, which was followed by pelvic floor ultrasound. Catheter could be removed after several weeks.

Mesh-related complications were defined by ICS/IUGA as foreign body reaction to the implant in terms of inflammation, infection and/or rejection, and the loss of effectivity over time [4]. The observed perioperative mesh-related complications correspond to the data of current meta-analyses and studies $[5,6]$. The study was within the range of the reported mesh-related complications. One case of apical prolapse recurrence can be referred to a possible avulsion of sacrospinal fixation and is not related to the mesh. PVDF offers good mechanical stability over 19 mos follow up and less complications compared to the available literature. Mesh erosion is reported to be about $10 \%$ after anterior mesh application [6]. In our study there were two cases of mesh exposure (5.9\%), which could be managed successfully. The patients with mesh exposure had several risk factors: current smoking status, menopause and adiposity. However, the complication rates were too low for multivariate analysis of risk factors [5]. No additional complications or intraoperative problems due to PVDF mesh were observed.

Other studies reported previously on mesh modifications by surface coating with collagen, titanium or absorbable polymers in animal and in vitro studies [27-30]. However, the results are inconsistent and study groups are small with few details on technical modifications. Some of these meshes have been now introduced into the market since as they were thought to be associated with lower complications. However, a standardised approach for market approval of an innovation or new device according to IDEAL-D system is needed. IDEAL demands structured and detailed description of the procedure and innovation steps. Doing this, the findings of studies are comparable for the researchers, bundle the evidence and prevent the repetition of same experiments. Different modifications of the surgical procedure over time are reported in accordance with early development IDEAL stage.

The limitations of the presented study is the small patient number and retrospective design, so that no statement on risk factors and the efficacy of surgical modifications for the outcome was possible. The absolute number of longterm complications as mesh exposure could be underestimated due to a follow up of less than five years. There could be a selection bias due to three patients lost to follow up. However, our study group was the first one to analyse the mesh modification according to IDEAL-criteria of surgical innovation [12]. Based on the preclinical results a monocentric feasibility and development trial with method modification is presented. This study presents as well postmarket-surveillance clinical data, as demanded by upcoming European medical device regulations. A consecutive Urogynecological registry for implants is currently under construction [31]. It is crucial that IDEAL-conform studies will be supported in the future. Therefore research funders need to recognize the nature of surgical innovation to encourage high-quality research approaches and improve the outcome for our patients.

Overall, the anterior PVDF-insertion for cystocele repair is safe and offers good functional results. The modification is implemented according to IDEAL-criteria of surgical innovations in a real-life setting. In reaction to FDA-reports on mesh associated problems, our international collaboration group present a unique 
implementation of IDEAL-system for mesh graft development in Urogynecology.

\section{Ethical approval}

Not needed due to retrospective design.

\section{Sources of funding}

No funding.

\section{Author contribution}

Dimitri Barski: Protocol development, data management, statistics, manuscript editing.

Christian Arndt and Holger Gerullis: Data collection and management, statistics.

Jin Yang and Mihaly Boros: Project development and proof reading.

Thomas Otto: Project development and proof reading.

Hans-Christian Kolberg: Protocol/project development, data management, manuscript editing.

\section{Conflict of interests}

The authors declare that there is no conflict of interests regarding the publication of this paper.

\section{Guarantor}

Dimitri Barski and Hans-Christian Kolberg.

\section{Research registration unique identifying number (UIN)}

Researchregistry2097.

\section{Acknowledgements}

All named authors meet the International Committee of Medical Journal Editors (ICMJE) criteria for authorship for this manuscript, take responsibility for the integrity of the work as a whole, and have given final approval to the version to be published. The authors highly appreciate the outstanding support throughout preclinical experiments from Albert Ramon, International Tissue Engineering Research Association (ITERA), Belgium.

\section{References}

[1] P.K. Amid, I.L. Lichtenstein, Current assessment of Lichtenstein tension-free hernia repair, Chirurg 68 (10) (1997) 959-964.

[2] T. Otto, B. Klosterhalfen, U. Klinge, M. Boros, D. Ysebaert, K. Williams, Implants in urogynecology, Biomed. Res. Int. 2015 (2015) 354342, http://dx.doi.org/ $10.1155 / 2015 / 354342$.

[3] FDA strengthens requirements for surgical mesh for the transvaginal repair of pelvic organ prolapse. http://www.fda.gov/NewsEvents/Newsroom/ PressAnnouncements/ucm479732.htm, Accessed 08 September 2016.

[4] B.T. Haylen, R.M. Freeman, S.E. Swift, et al., An International Urogynecological Association (IUGA)/International Continence Society (ICS)joint terminology and classification of the complications related directly to the insertion of prostheses (meshes, implants, tapes) \& grafts in female pelvic floor surgery, Int. Urogynecol. J. 22 (2011) 3-15, http://dx.doi.org/10.1007/s00192-0101324-9.

[5] D. Barski, T. Otto, H. Gerullis, Systematic review and classification of complications after anterior, posterior, apical, and total vaginal mesh implantation for prolapse repair, Surg. Technol. Int. 24 (2014) 217-224.

[6] C. Maher, B. Feiner, K. Baessler, C. Schmid, Surgical management of pelvic organ prolapse in women, Cochrane Database Syst. Rev. 4 (2013) CD004014, http://dx.doi.org/10.1002/14651858.CD004014.pub5.

[7] K. Ashok, E. Petri, Failures and complications in pelvic floor surgery, World J. Urol. 30 (4) (2012) 487-494, http://dx.doi.org/10.1007/s00345-011-0808-7.
[8] A. Gomelsky, R.R. Dmochowski, Biocompatibility assessment of synthetic sling materials for female stress urinary incontinence, J. Urol. 178 (4 Pt 1) (2007) 1171-1181, http://dx.doi.org/10.1016/j.juro.2007.05.123.

[9] H. Gerullis, E. Georgas, C. Eimer, et al., Evaluation of biocompatibility of alloplastic materials: development of a tissue culture in vitro test system, Surg. Technol. Int. XXI (2011) 21-27.

[10] H. Gerullis, E. Georgas, M. Borós, et al., Inflammatory reaction as determinant of foreign body reaction is an early and susceptible event after mesh implantation, Biomed. Res. Int. 2014 (2014) 510807, http://dx.doi.org/10.1155/ 2014/510807.

[11] H. Gerullis, B. Klosterhalfen, M. Borós, et al., IDEAL in meshes for prolapse, urinary incontinence, and hernia repair, Surg. Innov. 20 (5) (2013) 502-508, http://dx.doi.org/10.1177/1553350612472987.

[12] P. McCulloch, D.G. Altman, W.B. Campbell, et al., No surgical innovation without evaluation: the IDEAL recommendations, Lancet 374 (9695) (2009) 1105-1112, http://dx.doi.org/10.1016/S0140-6736(09)61116-8.

[13] A. Sedrakyan, B. Campbell, J.G. Merino, R. Kuntz, A. Hirst, P. McCulloch, IDEAL$\mathrm{D}$ : a rational framework for evaluating and regulating the use of medical devices, BMJ 353 (2016) i2372.

[14] B.T. Haylen, C.F. Maher, M.D. Barber, et al., An International Urogynecological Association (IUGA)/International Continence Society (ICS) joint report on the terminology for female pelvic organ prolapse (POP), Int. Urogynecol. J. 27 (4) (2016) 655-684, http://dx.doi.org/10.1007/s00192-016-3003-y.

[15] P.A. Clavien, J. Barkun, M.L. de Oliveira, et al., The Clavien-Dindo classification of surgical complications: five-year experience, Ann. Surg. 250 (2) (2009) 187-196, http://dx.doi.org/10.1097/SLA.0b013e3181b13ca2.

[16] S. Srikrishna, D. Robinson, L. Cardozo, Validation of the patient global impression of improvement (PGI-I) for urogenital prolapse, Int. Urogynecol. J. 21 (5) (2010) 523-528, http://dx.doi.org/10.1007/s00192-009-1069-5.

[17] L. Mearini, A. Zucchi, E. Nunzi, M. Di Biase, V. Bini, E. Costantini, The S.A.C.S (Satisfaction-Anatomy-Continence-Safety) score for evaluating pelvic organ prolapse surgery: a proposal for an outcome-based scoring system, Int. Urogynecol. J. 26 (7) (2015) 1061-1067, http://dx.doi.org/10.1007/s00192-0152655-3.

[18] U. Klinge, B. Klosterhalfen, Modified classification of surgical meshes for hernia repair based on the analyses of 1,000 explanted meshes, Hernia 16 (3) (2012) 251-258, http://dx.doi.org/10.1007/s10029-012-0913-6.

[19] U. Klinge, B. Klosterhalfen, A.P. Ottinger, K. Junge, V. Schumpelick, PVDF as a new polymer for the construction of surgical meshes, Biomaterials 23 (16) (2002) 3487-3493.

[20] E. Urban, M.W. King, R. Guidoin, et al., Why make monofilament sutures out of polyvinylidene fluoride? ASAIO J. 40 (2) (1994) 145-156.

[21] A. Verbo, P. Pafundi, A. Manno, et al., Polyvinylidene fluoride mesh (PVDF, DynaMesh ${ }^{\mathbb{B}}$-IPOM) in the laparoscopic treatment of incisional hernia: a prospective comparative trial versus Gore ${ }^{(\mathbb{R}}$ ePTFE DUALMESH ${ }^{\mathbb{B}}$ plus, Surg. Technol. Int. 28 (2016) 147-151.

[22] T. Sommer, H. Friis-Andersen, DynaMesh ${ }^{\circledR}$ in the repair of laparoscopic ventral hernia: a prospective trial, Hernia 17 (5) (2013) 613-618, http://dx.doi.org/ 10.1007/s10029-013-1090-y.

[23] R. Joukhadar, G. Meyberg-Solomayer, A. Hamza, et al., A novel operative procedure for pelvic organ prolapse utilizing a MRI-visible mesh implant: safety and outcome of modified laparoscopic bilateral sacropexy, Biomed. Res. Int. 2015 (2015) 860784, http://dx.doi.org/10.1155/2015/860784.

[24] K.G. Noé, S. Schiermeier, I. Alkatout, M. Anapolski, Laparoscopic pectopexy: a prospective, randomized, comparative clinical trial of standard laparoscopic sacral colpocervicopexy with the new laparoscopic pectopexy-postoperative results and intermediate-term follow-up in a pilot study, J. Endourol. 29 (2) (2015) 210-215, http://dx.doi.org/10.1089/end.2014.0413.

[25] D. Altman, T. Väyrynen, M.E. Engh, et al., Anterior colporrhaphy versus transvaginal mesh for pelvic-organ prolapse, N. Engl. J. Med. 364 (2011) 1826, http://dx.doi.org/10.1056/NEJMoa1009521.

[26] A. Gild, B. Schoenfisch, M. Huebner, S. Brucker, D. Wallwiener, C. Reisenauer, Does applying postoperative suprapubic catheterisation in urogynecology benefit patients? Arch. Gynecol. Obstet. 293 (5) (2016) 1039-1042, http:// dx.doi.org/10.1007/s00404-015-3928-2.

[27] G. Böhm, Y. Ushakova, H.P. Alizai, et al., Biocompatibility of PLGA/sP(EO-statPO)-coated mesh surfaces under constant shearing stress, Eur. Surg. Res. 47 (3) (2011) 118-129, http://dx.doi.org/10.1159/000329412.

[28] K. Junge, R. Rosch, U. Klinge, et al., Titanium coating of a polypropylene mesh for hernia repair: effect on biocompatibility, Hernia 9 (2) (2005) 115-119, http://dx.doi.org/10.1007/s10029-004-0292-8.

[29] A. Lukasiewicz, J. Skopinska-Wisniewska, A. Marszalek, S. Molski, T. Drewa, Collagen/Polypropylene composite mesh biocompatibility in abdominal wall reconstruction, Plast. Reconstr. Surg. 131 (5) (2013) 731e-740e, http:// dx.doi.org/10.1097/PRS.0b013e3182865d2c.

[30] A. Mangera, A.J. Bullock, S. Roman, C.R. Chapple, S. MacNeil, Comparison of candidate scaffolds for tissue engineering for stress urinary incontinence and pelvic organ prolapse repair, BJU Int. 112 (5) (2013) 674-685, http:// dx.doi.org/10.1111/bju.12186.

[31] D. Barski, H. Gerullis, T. Ecke, et al., Development of an online platform for registration and outcome measurement of urogynecological implants according to IDEAL-system, Int. J. Surg. 36 (2016) 141-142, http://dx.doi.org/ 10.1016/j.ijsu.2016.11.048. 